\title{
Actividad antioxidante, compuestos fenólicos y ácido ascórbico de frutillas en dos sistemas de producción
}

\author{
Rufino Fernando F Cantillano'; Julia Maria M Ávila²; Maria do Carmo R Peralba ${ }^{3}$; Tânia Mara \\ Pizzolato $^{3}$; Ricardo P Toralles ${ }^{2}$ \\ ${ }^{1}$ Embrapa Clima Temperado, C. Postal 403, 96010-971 Pelotas-RS; fernando.cantillano@cpact.embrapa.br; ${ }^{2}$ IFSUL-rio-grandense, \\ Depto. Química, Praça 20 de setembro 455, Centro, 96015-360 Pelotas-RS; julia.avila@ufrgs.br; toralles@pelotas.ifsu.edu.br; ${ }^{3}$ UFRGS, \\ Depto. Química Inorganica-Inst. Química, Campus do Vale, Av. Bento Gonçalves 9500 Agronomia, Porto Alegre-RS; mcarmo@iq.ufrgs. \\ br; taniamar@iq.ufrgs.br
}

\section{RESUMEN}

El objetivo de este trabajo fue determinar la influencia del sistema de producción y del período de almacenamiento de frutillas sobre la pérdida de masa, compuestos fenólicos totales, antocianinas totales, ácido ascórbico (vitamina $\mathrm{C}$ ), actividad antioxidante y sus correlaciones. En este experimento fueron utilizadas frutillas 'Camarosa' y 'Camino Real' con madurez comercial, procedentes de cultivos en sistemas de producción orgánico y convencional, localizados en el municipio de Pelotas-RS. Los frutos fueron almacenados durante $0,2,5$ y 8 días a $1^{\circ} \mathrm{C}$ y $90-95 \%$ de HR. En general, el contenido de fenoles totales, de antocianinas totales y la actividad antioxidante aumentaron durante el almacenamiento refrigerado, mientras que los contenidos de ácido ascórbico y de masa fresca disminuyeron significativamente. Los contenidos de fenoles totales y de antocianinas totales presentaron correlación positiva con la actividad antioxidante, siendo la correlación con el contenido de ácido ascórbico negativa. Las frutillas producidas en sistema orgánico y conservadas durante cinco días, mostraron mayores valores de actividad antioxidante. Después de ocho días de almacenamiento a $1{ }^{\circ} \mathrm{C}$, los frutos del sistema de producción orgánico presentaron menor degradación de antocianinas y de ácido ascórbico que los frutos del sistema de producción convencional.

Palabras-clave: Fragaria x ananassa, postcosecha, sistema de producción orgánico, calidad de la fruta.

\section{ABSTRACT}

Antioxidant activity, phenolic compounds and ascorbic acid content in strawberries from two crop production systems

The aim of this study was to determine the influence of the crop production system and storage period on weight loss, total phenolic content, total anthocyanin content, ascorbic acid (vitamin C) content, and antioxidant activity in strawberries, as well as their correlations. For this experiment, strawberry fruits of 'Camarosa' and 'Camino Real' at commercial maturity from both organic and conventional crop production systems located in Pelotas, Brazil, were used. The fruits were cold stored for $0,2,5$, and 8 days at $1{ }^{\circ} \mathrm{C}$ and $90-95 \% \mathrm{RH}$. In general the total phenolic, total anthocyanin levels and antioxidant activity increased during cold storage. Ascorbic acid levels and fresh weight decreased significantly. Total phenol and anthocyanin levels showed positive correlations with total antioxidant activity, while the ascorbic acid content showed a negative correlation. Strawberries produced by the organic system and cold stored for five days showed higher values of antioxidant activity. After storage at $1^{\circ} \mathrm{C}$ for eight days, strawberry fruits from the organic production system presented lesser anthocyanin and ascorbic acid degradation than the fruits from the conventional production system.

Keywords: Fragaria x ananassa, postharvest, organic production system, fruit quality.

(Recebido para publicação em 1 de dezembro de 2011; aceito em 24 de julho de 2012) (Received on December 1, 2011; accepted on July 24, 2012)

\begin{abstract}
A ctualmente, la producción y el consumo de alimentos sanos, especialmente de frutas producidas con clara conciencia ecológica representan una oportunidad viable para la agricultura. El crecimiento acelerado de la demanda por este tipo de producto, refleja un cambio en las preferencias de los consumidores, por alimentos inocuos, nutritivos y generados sin técnicas agresivas al medio ambiente (Cantillano, 2008). En este contexto, el sistema de producción orgánico viene a suplir la demanda del mercado, debido a que no hace uso de productos químicos sintéticos, tales como fertilizantes y pesticidas, no contaminando el medio
\end{abstract}

ambiente y sin colocar en riesgo la salud de los consumidores.

La frutilla (Fragaria x ananassa) es un fruto muy apreciado y comercializado mundialmente, siendo que Brasil posee una gran producción y consumo. Los principales productores son los estados de Minas Gerais, São Paulo y Rio Grande do Sul, cuya producción se destina a atender el mercado in natura y la industria en la forma de jugos, jaleas, mermeladas y helados (Seeram et al., 2006; Calvete et al., 2008). Pero, contaminaciones de frutas con pesticidas están dejando inseguro al consumidor. De esta manera, investigaciones con sistemas orgánicos de producción están siendo realizadas (Castro et al., 2003).

La frutilla es un fruto muy perecedero, con alta tasa respiratoria y de corta vida postcosecha. Los daños mecánicos, heridas y golpes durante la cosecha, transporte y comercialización dejan el fruto susceptible al ataque de microorganismos, causando pérdidas nutritivas, cualitativas y económicas. El almacenamiento refrigerado es el método más utilizado para mantener la calidad de los frutos in natura. Las condiciones recomendadas para el almacenamiento de frutillas varían de 0 a $1^{\circ} \mathrm{C}$, con $90-95 \%$ de humedad relativa (Cantillano et al., 2008). 
La calidad de las frutillas está condicionada por factores de precosecha y postcosecha. Así, tratamientos culturales como fertilización, aplicación de pesticidas, calidad de plántulas, condiciones meteorológicas y disponibilidad de agua son factores de precosecha importantes para obtener un producto de buena calidad. Entre los factores de postcosecha mas relevantes pueden ser citados el grado de madurez a la cosecha, manejo cuidadoso de la fruta, temperatura y humedad relativa adecuadas y sin fluctuaciones durante el almacenamiento refrigerado. Sin embargo, existen pocas informaciones sobre el papel de cada uno de estos factores en la pérdida de calidad de la frutilla, principalmente cuando se comparan los sistemas de producción convencional y orgánico. Un estudio realizado con los cultivares Diamante, San Juan y Lanai demostró que frutillas cultivadas en un sistema orgánico presentaron mayor actividad antioxidante, concentración de compuestos fenólicos y vitamina $\mathrm{C}$ (Reganold et al., 2010). Terrazzan et al. (2006) obtuvieron resultados semejantes para vitamina $\mathrm{C}$, en frutillas cultivar Oso Grande, en un sistema de producción orgánico.

Existen numerosos trabajos relacionando factores de pre y postcosecha con la calidad de frutillas en sistemas de producción convencional (Kalt et al., 1999; Ayala-Zavala et al., 2004; Malgarim et al., 2006; Cheel et al., 2007). Sin embargo, no existen relatos de experimentos similares, abordando los cultivares Camarosa y Camino Real cultivados en sistemas de producción convencional y orgánico. Entre los factores de calidad que pueden ser alterados, pueden ser mencionados la pérdida de masa fresca, el color de la epidermis, la firmeza del fruto, el sabor, el aroma, el nivel de ácido ascórbico, los compuestos fenólicos y las características sensoriales (PelayoZaldívar et al., 2005; Terrazzan et al., 2006).

La frutilla también posee varios compuestos bioactivos y elevada actividad antioxidante, que pueden conferir importantes beneficios a la salud humana (Ferreyra et al., 2007).
Estudios muestran que el consumo de frutillas in natura o en forma de jugo inhibe bacterias patógenas, tales como Salmonella y Staphylococcus (Badjakov et al., 2008), presenta acción anticarcinógena (Olsson et al., 2007), anticoagulante (Torres et al., 2008) y reduce enfermedades cardiovasculares (Erlund et al., 2008).

El objetivo del presente trabajo fue estudiar la influencia del período de almacenamiento refrigerado y del sistema de producción en la pérdida de masa y en los niveles de fenoles totales, antocianinas totales, ácido ascórbico y en la actividad antioxidante en frutillas de los cultivares Camarosa y Camino Real.

\section{MATERIAL Y MÉTODOS}

Se utilizaron frutillas (Fragaria $\mathrm{x}$ ananassa) cultivadas en el año 2008 de los cultivares Camarosa y Camino Real producidas en sistemas convencional y orgánico (Camino Real Convencional $=$ CRC; Camino Real Orgánico $=$ $\mathrm{CRO}$; Camarosa Convencional $=\mathrm{CC}$; Camarosa Orgánico $=\mathrm{CO}$ ) en una finca comercial localizada en Gama, Monte Bonito, $9^{\circ}$ distrito del municipio de Pelotas (32 $52^{\prime}$ 'S, $52^{\circ} 21^{\prime} \mathrm{O}$, altitud 220 $\mathrm{m})$, en suelo clasificado como arcilloso rojo-amarillo distrófico (Streck et al., 2008). Las frutillas de los sistemas convencional y orgánico fueron cultivadas sobre un film de plástico negro mulching y riego por goteo. La plantación fue realizada en un ambiente protegido tipo túnel bajo, con distancias de 30 x $30 \mathrm{~cm}$ y cada parcela compuesta por tres hileras. La fertilización para el sistema convencional fue NPK (5-3015) y para el orgánico se utilizó abono orgánico con estiércol de aves y de ganado.

Fueron cosechados aleatoriamente 7 $\mathrm{kg}$ de frutos de cada cultivar en los dos sistemas de producción, sin defectos $\mathrm{y}$ de aspecto maduro, superficie de color rojo-brillante entre $50 \%-75 \%$ (Cantillano et al., 2003). De estas muestras fueron seleccionados 15 frutos con tres repeticiones, los que se almacenaron durante $0,2,5$ y 8 días en cámara fría a $1 \pm 0,5^{\circ} \mathrm{C}$ y $90-95 \%$ de HR. En la cosecha y después del almacenamiento, los análisis fueron realizados en los laboratorios de Postcosecha y Tecnología de Alimentos de la Embrapa Clima Temperado y de Alimentos de IFSUL (Campus Pelotas). La variable pérdida de masa fue calculada mediante la diferencia entre el peso inicial de los frutos (balanza Marte AS5500-precisión de $\pm 0,01 \mathrm{~g})$ y aquel obtenido para cada período de almacenamiento, siendo el resultado expresado en porcentaje.

Los análisis en triplicado fueron realizados con el jugo del fruto obtenido por trituración de la pulpa molida en centrífuga (Wallita).

El nivel de fenoles totales (FT) se determinó según el método de Singleton \& Rossi (1965) y los resultados se expresaron en microgramos de ácido gálico (equivalentes de ácido gálico$\mathrm{GAE})$ por gramo de fruto, $\mu \mathrm{g} \mathrm{GAE} \mathrm{g}^{-1}$.

El nivel de ácido L-ascórbico se determinó según la AOAC (2000), utilizando el método titulométrico con el uso de 2,6-diclorofenolindofenol a $0,01 \%$. Los resultados se expresaron en $\mathrm{mg}$ de ácido ascórbico en $100 \mathrm{~g}$ de fruto.

El nivel de antocianinas totales (ANT) se determinó por el método de Lees \& Francis (1972) y los resultados se expresaron en mg de antocianinas representada en cianidina-3-glicosidio en $100 \mathrm{~g}$ de fruto. La actividad antioxidante relativa en 30 minutos se determinó según el método de Ozgen et al. (2006), por la reducción del radical estable DPPH, a través de la acción de los antioxidantes presentes en la muestra. Los resultados se expresaron en micro moles $(\mu \mathrm{M})$ de actividad antioxidante equivalente a Trolox (TEAC) por gramo de fruto.

El diseño experimental fue completamente al azar, con arreglo factorial de $4 \times 2 \times 2$. La unidad experimental fue de 15 frutos con 3 repeticiones. La pérdida de masa durante el almacenamiento fue corregida para todas las variables estudiadas. Los valores porcentuales se transformaron en arcoseno $\sqrt{\mathrm{x}}$ previo a los análisis. Los datos fueron sometidos a análisis de varianza y los promedios comparados por la prueba de rango múltiple de Tukey $(p \leq 0,05)$, siendo utilizado el programa STATISTICA (Statsoft, 1996). También 
fueron calculados los coeficientes de regresión linear.

\section{RESULTADOS Y DISCUSIÓN}

Los resultados de pérdida de masa fresca, niveles de fenoles totales, antocianinas totales, ácido ascórbico y actividad antioxidante relativa para los cultivares Camino Real y Camarosa cultivados en el sistema convencional y orgánico son mostrados en la Tabla 1. Se observó una pérdida de masa fresca significativa $(p \leq 0,05)$ y creciente en los ocho días de almacenamiento, siendo que la mayor pérdida fue observada en el cultivar Camino Real producido en el sistema orgánico $(11,21 \%)$, después de ocho días de almacenamiento. El retraso de la pérdida de masa en los cultivares del sistema convencional, puede estar relacionado al uso de fungicidas en el cultivo (Mazaro et al., 2008). Nunes et al. (2005) relataron pérdida de $11 \%$ en frutos del cultivar Oso Grande almacenados por ocho días a $1^{\circ} \mathrm{C}$. Según Hernández-Muñoz et al. (2006), la pérdida de masa fresca superior a $10 \%$ es un indicador de pérdida de calidad en frutillas, siendo el producto rechazado por los consumidores.

Los niveles de fenoles totales, de modo general, aumentaron significativamente hasta el quinto día de almacenamiento, para los dos cultivares en ambos sistemas de producción (Tabla 1). El cultivar Camino Real en el sistema convencional presentó niveles de $1.422,94 \mu \mathrm{g} \mathrm{g}^{-1}$ significativamente superior a los demás $(\mathrm{p} \leq 0,05)$. Estos valores son mayores a los relatados por Silva (2007), para los mismos cultivares, en frutos producidos en el sistema convencional, durante la temporada
2005/2006.

Después de ocho días de almacenamiento a $1^{\circ} \mathrm{C}$, los niveles de fenoles totales en los frutos del cultivar Camino Real tuvieron aumento superior a $14 \%$, en relación al día cero de almacenamiento, mientras que los frutos del cultivar Camarosa presentaron un aumento levemente superior a $8 \%$ (Figura 1a). Ayala-Zavala et al. (2004), estudiando el efecto de la temperatura de almacenamiento en los frutos del cultivar Chandler, observaron que el nivel de fenoles totales se mantuvo prácticamente constante a $0^{\circ} \mathrm{C}$ por 13 días (650 mg $100^{-1}$ de fruto fresco), sin embargo un aumento substancial fue observado a $10^{\circ} \mathrm{C}\left(1050 \mathrm{mg} 100^{-1} \mathrm{de}\right.$ fruto fresco). Además de la temperatura de almacenamiento, el aumento en el nivel de estos compuestos se puede deber al grado de estrés mecánico y

Tabla 1. Características físicas y químicas de frutillas de los cultivares Camarosa y Camino Real producidas en sistema de producción convencional y orgánico y almacenadas durante ocho días a $1{ }^{\circ} \mathrm{C}$ y $90-95 \%$ HR (physical and chemical characteristics of Camarosa and Camino Real strawberry cultivars produced in conventional and organic production systems and stored during eight days at $1{ }^{\circ} \mathrm{C}$ and $90-95 \%$ RH). Pelotas, Embrapa Clima Temperado/IFSUL, 2008.

\begin{tabular}{|c|c|c|c|c|c|c|c|}
\hline \multirow{2}{*}{ Características } & \multirow{2}{*}{$\begin{array}{l}\text { Sistema de } \\
\text { producción }\end{array}$} & \multirow{2}{*}{ Cultivar } & \multicolumn{4}{|c|}{ Almacenamiento (días)* } & \multirow{2}{*}{$\begin{array}{l}\mathrm{CV} \\
(\%)\end{array}$} \\
\hline & & & $\mathbf{0}$ & 2 & 5 & 8 & \\
\hline \multirow{4}{*}{$\begin{array}{l}\text { Pérdida de masa } \\
(\%)\end{array}$} & \multirow{2}{*}{ Convencional } & Camino Real & $0,00 \mathrm{aC}$ & $2,32 \mathrm{~dB}$ & $2,70 \mathrm{cB}$ & $8,93 \mathrm{bA}$ & 3,51 \\
\hline & & Camarosa & $0,00 \mathrm{aC}$ & $2,45 \mathrm{cB}$ & $2,60 \mathrm{cB}$ & $9,49 \mathrm{bA}$ & 2,69 \\
\hline & \multirow{2}{*}{ Orgánico } & Camino Real & $0,00 \mathrm{aD}$ & $3,94 \mathrm{aB}$ & $3,27 \mathrm{aC}$ & 11,21 aA & 1,70 \\
\hline & & Camarosa & $0,00 \mathrm{aC}$ & $2,77 \mathrm{bB}$ & $3,22 \mathrm{bB}$ & $9,70 \mathrm{bA}$ & 2,81 \\
\hline \multirow{4}{*}{$\begin{array}{l}\text { Fenoles totales } \\
(\mu \mathrm{g} \mathrm{GAE} / \mathrm{g})\end{array}$} & \multirow{2}{*}{ Convencional } & Camino Real & $1182,88 \mathrm{cC}$ & $1326,34 \mathrm{aB}$ & 1422,94 aA & $1401,50 \mathrm{aA}$ & 1,24 \\
\hline & & Camarosa & 1273,48 aB & 1351,02 aAB & $1368,53 \mathrm{cA}$ & $1381,32 \mathrm{bA}$ & 2,00 \\
\hline & \multirow{2}{*}{ Orgánico } & Camino Real & $1130,67 \mathrm{~dB}$ & 1202,88 сAB & 1209,82 dBA & $1299,94 \mathrm{cA}$ & 3,31 \\
\hline & & Camarosa & $1250,86 \mathrm{bB}$ & $1257,40 \mathrm{cB}$ & $1391,74 \mathrm{bA}$ & $1375,56 \mathrm{bA}$ & 3,42 \\
\hline \multirow{4}{*}{$\begin{array}{l}\text { Antocianinas totales } \\
(\mathrm{mg} / 100 \mathrm{~g})\end{array}$} & \multirow{2}{*}{ Convencional } & Camino Real & 24,15 aA & $25,93 \mathrm{aA}$ & $26,22 \mathrm{aA}$ & $21,09 \mathrm{bB}$ & 4,00 \\
\hline & & Camarosa & $17,78 \mathrm{bB}$ & $18,41 \mathrm{bB}$ & $22,35 \mathrm{bA}$ & $17,02 \mathrm{cB}$ & 5,41 \\
\hline & \multirow{2}{*}{ Orgánico } & Camino Real & $16,57 \mathrm{cB}$ & $17,75 \mathrm{cB}$ & $21,12 \mathrm{cA}$ & $21,70 \mathrm{aA}$ & 6,19 \\
\hline & & Camarosa & $11,93 \mathrm{~dB}$ & $16,92 \mathrm{cA}$ & $17,36 \mathrm{dA}$ & $17,65 \mathrm{cA}$ & 4,53 \\
\hline \multirow{4}{*}{$\begin{array}{l}\text { Ácido ascórbico } \\
(\mathrm{mg} / 100 \mathrm{~g})\end{array}$} & \multirow{2}{*}{ Convencional } & Camino Real & 55,56 a $\mathrm{A}$ & $41,54 \mathrm{cB}$ & $40,01 \mathrm{bBC}$ & $36,05 \mathrm{bC}$ & 3,77 \\
\hline & & Camarosa & $53,50 \mathrm{bA}$ & $46,22 \mathrm{aB}$ & $39,01 \mathrm{cC}$ & $34,58 \mathrm{cC}$ & 4,75 \\
\hline & \multirow{2}{*}{ Orgánico } & Camino Real & $52,00 \mathrm{cA}$ & $42,65 \mathrm{cB}$ & $43,66 \mathrm{aB}$ & $34,05 \mathrm{cC}$ & 5,63 \\
\hline & & Camarosa & $47,68 \mathrm{dA}$ & $43,46 \mathrm{bA}$ & 44,52 aA & $36,68 \mathrm{aB}$ & 5,29 \\
\hline \multirow{4}{*}{$\begin{array}{l}\text { Actividad } \\
\text { antioxidante } \\
(\mathrm{TEAC}-\mathrm{DPPH}) \\
(\mu \mathrm{M} \mathrm{TE} / \mathrm{g})\end{array}$} & \multirow{2}{*}{ Convencional } & Camino Real & $4,62 \mathrm{cB}$ & 4,92 dAB & $5,36 \mathrm{dA}$ & 4,93 aAB & 4,49 \\
\hline & & Camarosa & $4,69 \mathrm{bC}$ & 5,52 aAB & $5,81 \mathrm{cA}$ & $4,90 \mathrm{aBC}$ & $\begin{array}{r}4,34 \\
\ldots \ldots\end{array}$ \\
\hline & \multirow{2}{*}{ Orgánico } & Camino Real & $4,67 \mathrm{bB}$ & $5,00 \mathrm{cB}$ & $6,14 \mathrm{bA}$ & $4,47 \mathrm{bB}$ & 6,88 \\
\hline & & Camarosa & $5,07 \mathrm{aB}$ & $5,36 \mathrm{bB}$ & $6,24 \mathrm{aA}$ & $4,89 \mathrm{aB}$ & 2,88 \\
\hline
\end{tabular}

*Promedios seguidos de la misma letra minúscula en la columna o mayúscula en la línea no difieren estadísticamente entre sí por la prueba de rango múltiple de Tukey $(\mathrm{p} \leq 0,05)$; los datos fueron corregidos con relación a la pérdida de masa (*means followed by the same small letter in the column or capital letter in the row do not differ statistically from each other by the Tukey ( $\mathrm{p} \leq 0.05)$ multiple range test; data were corrected in relation to the mass loss). 


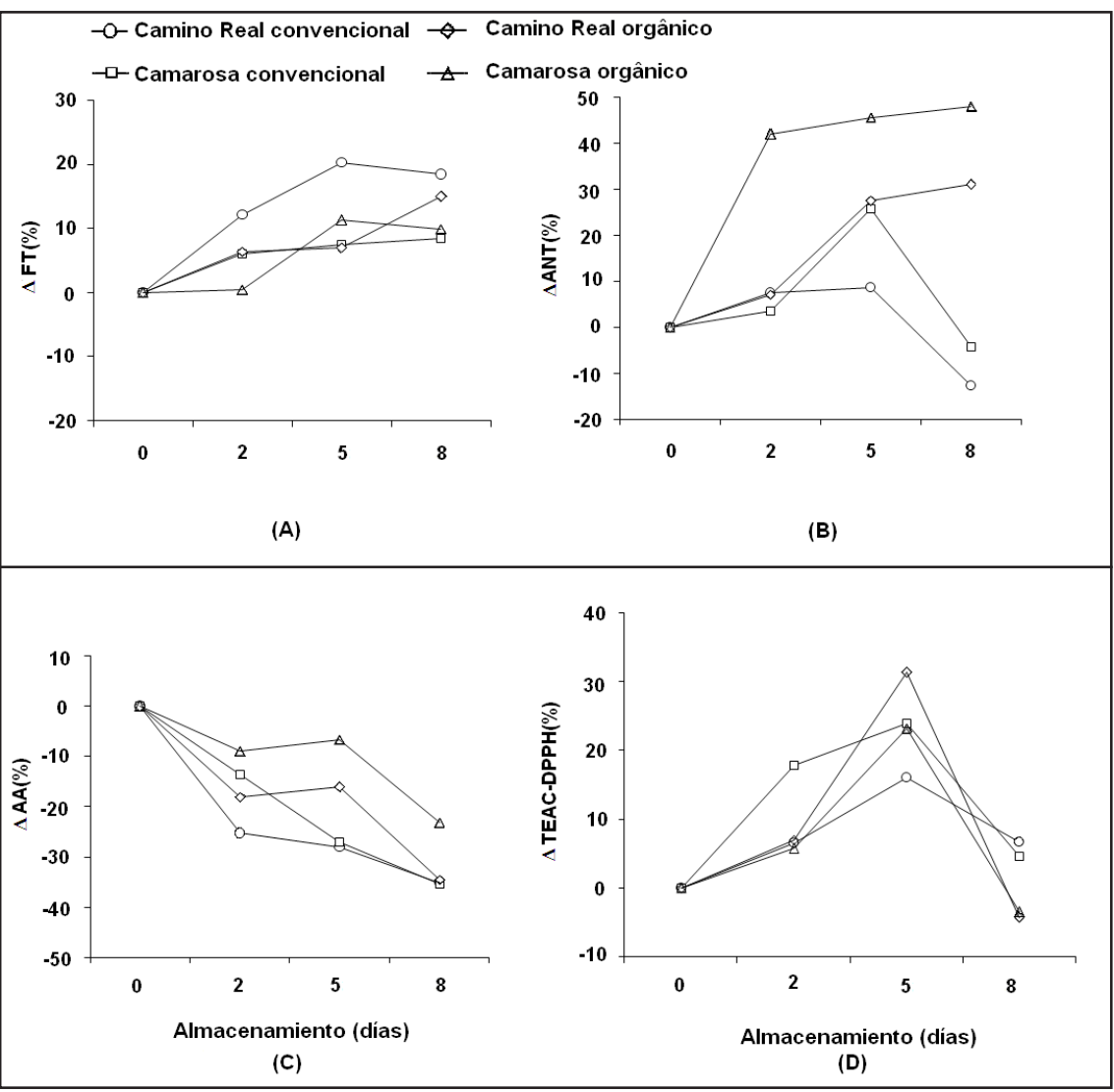

Figura 1. Variación porcentual durante el período de almacenamiento en frutillas 'Camarosa' y 'Camino Real' almacenadas a $1^{\circ} \mathrm{C}$ y $90-95 \% \mathrm{HR}$, de los niveles de fenoles totales- $\triangle \mathrm{FT}$ (A); antocianinas totales- $\triangle \mathrm{ANT}(\mathrm{B})$; ácido ascórbico- $\triangle \mathrm{AA}(\mathrm{C})$ y actividad antioxidante- $\triangle \mathrm{TEAC}$ DPPH (D); Cada variable fue calculada en relación al tiempo cero de almacenamiento (percentage change during the storage period in 'Camarosa' and 'Camino Real' strawberry fruits stored at $1^{\circ} \mathrm{C}$ and $90-95 \% \mathrm{RH}$, of total phenol levels $\triangle \mathrm{FT}(\mathrm{A})$; total anthocyanins- $\triangle \mathrm{ANT}$ (B); ascorbic acid- $\triangle \mathrm{AA}(\mathrm{C})$ and antioxidant activity- $\triangle \mathrm{TEAC}-\mathrm{DPPH}(\mathrm{D})$. Each variable was calculated in relation to the zero storage time) ( $\Delta=$ variación). Pelotas, Embrapa Clima Temperado/IFSUL, 2008.

Tabla 2. Coeficientes de correlación (r) entre la actividad antioxidante (TEAC-DPPH) y los niveles de fenoles totales, antocianinas totales y ácido ascórbico, para frutillas 'Camarosa' y 'Camino Real' producidas en sistemas de producción convencional y orgánico (correlation coefficients (r) between antioxidant activity (TEAC-DPPH) and total phenol levels, total anthocyanins and ascorbic acid for 'Camarosa' and 'Camino Real' strawberries produced in conventional and organic production systems). Pelotas, Embrapa Clima Temperado/ IFSUL, 2008.

\begin{tabular}{lcccc}
\hline & \multicolumn{4}{c}{ Actividad antioxidante (coeficiente de correlación, r) } \\
\cline { 2 - 5 } & $\mathbf{C R C}$ & $\mathbf{C C}$ & $\mathbf{C R O}$ & $\mathbf{C O}$ \\
\hline Fenoles totales & $0,79^{*}$ & $0,87^{*}$ & $0,72^{*}$ & $0,79^{*}$ \\
Antocianinas totales & $0,82^{*}$ & $0,66^{*}$ & $0,84^{*}$ & $0,69^{*}$ \\
Ácido ascórbico & $-0,82^{*}$ & $-0,81^{*}$ & $-0,29^{\mathrm{ns}}$ & $-0,41^{\mathrm{ns}}$ \\
\hline
\end{tabular}

*Significativo $(\mathrm{p} \leq 0,05)$; $\mathrm{ns}=$ no significativo; $\mathrm{CRC}=$ Camino Real Convencional; $\mathrm{CC}=$ Camarosa Convencional; $\mathrm{CRO}=$ Camino Real Orgánico; $\mathrm{CO}=$ Camarosa Orgánico (*significant $(\mathrm{p} \leq 0,05) ; \mathrm{ns}=$ not significant; $\mathrm{CRC}=$ Camino Real Conventional; $\mathrm{CC}=$ Camarosa Conventional; $\mathrm{CRO}=$ Camino Real Organic; $\mathrm{CO}=$ Camarosa Organic).

biológico, a la exposición a la luz y a la disponibilidad de oxígeno (Crozier et al., 2000; Naczk \& Shahidi, 2004).

El tiempo de almacenamiento y frutos cultivados en el sistema orgánico, mientras que en los cultivados en el sistema convencional, disminuyeron en el $8^{\circ}$ día de almacenamiento (Tabla 1). El cultivar Camino Real presentó un mayor nivel de antocianinas $\left(26,22 \mathrm{mg} \mathrm{100^{-1 } )}\right.$ en el $5^{\circ}$ día de almacenamiento, cuando cultivado en el sistema convencional y en el $8^{\circ}$ día cuando cultivado en el sistema orgánico. Ayala-Zavala et al. (2004) encontraron $28 \mathrm{mg} 100$ $\mathrm{g}^{-1}$ en frutos del cultivar Chandler almacenados a $0^{\circ} \mathrm{C}$ por cinco días, valor próximo al encontrado para el cultivar Camino Real producido en el sistema convencional. Después de ocho días de almacenamiento, en los frutos cultivados en el sistema de producción orgánico, el aumento de antocianinas fue superior a $30 \%$, mientras que en el convencional se observó una pérdida superior a 4\% (Figura 1b). Un análisis comparativo entre el $5^{\circ}$ y $8^{\circ}$ día indica que el sistema de producción orgánico preserva mejor las antocianinas que el convencional. Holcroft \& Kader (1999), en frutos del cultivar Selva almacenados a $5^{\circ} \mathrm{C}$ por 10 días, también observaron una ganancia significativa de $35 \%$ en los niveles de antocianinas.

Los resultados encontrados para fenoles y antocianinas indican la síntesis de estos compuestos durante el período de almacenamiento, lo que puede deberse a la combinación de precursores derivados de las vías del shiquimato y del acetato (Winkel-Shirley, 2001).

Los niveles de ácido ascórbico disminuyeron durante el almacenamiento de las frutillas en ambos sistemas de producción. Para el $8^{\circ}$ día, los niveles en frutos del cultivar Camarosa convencional $\left(34,6 \mathrm{mg} 100 \mathrm{~mL}^{-1}\right)$ y Camino Real orgánico $(34,0 \mathrm{mg}$ $100 \mathrm{~mL}^{-1}$ ) fueron significativamente inferiores $(\mathrm{p} \leq 0,05)$ a los demás, para esta vitamina (Tabla 1). Krolow et al. (2007) relataron para frutos del cultivar Aromas producidos en sistema convencional y orgánico, 56,50 y 52,08 $\mathrm{mg} 100 \mathrm{~mL}^{-1}$, respectivamente. Terrazzan et al. (2006), relataron mayores valores de vitamina $C$ en los frutos del cultivar Oso Grande producidos en sistema orgánico. La disminución de esta vitamina fue superior a $35 \%$ en sistema convencional, para ambos cultivares 


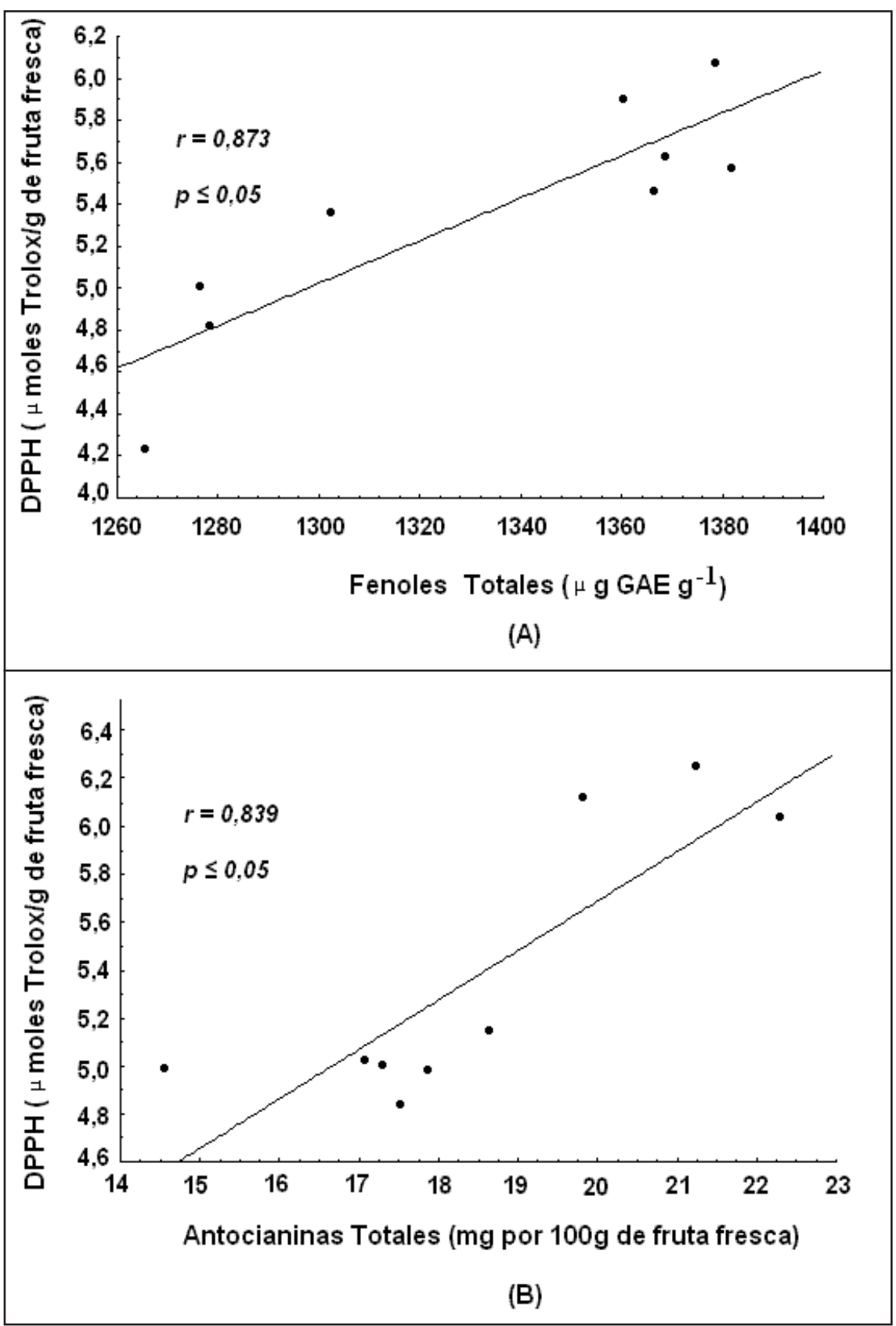

Figura 2. Correlación entre la actividad antioxidante (DPPH) y los niveles de fenoles totales para frutillas del cultivar Camarosa en sistema convencional (A) y correlación entre DPPH y antocianinas totales para frutillas del cultivar Camino Real en sistema orgánico (B) (correlation between antioxidant activity (DPPH) and total phenol levels for Camarosa strawberries cultivar in conventional system (A) and correlation between DPPH and total anthocyanins for Camino Real strawberries cultivar in organic system (B)). Pelotas, Embrapa Clima Temperado/IFSUL, 2008.

(Figura 1c), mientras que en sistema de producción orgánico, esta reducción fue menor para el cultivar Camarosa (23\%). Según Nunes et al. (1998), la disminución de la vitamina $\mathrm{C}$ está asociada a la pérdida de agua, que causa la oxidación de la misma. Otros autores también observaron la pérdida de esta vitamina durante el almacenamiento (Malgarim et al., 2006; Cantillano et al., 2008).

Los datos de actividad antioxidante relativa (Tabla 1) revelaron aumento para los cultivares Camino Real y Camarosa cultivados en ambos sistemas, hasta el $5^{\circ}$ día de almacenamiento.
En ese período, los frutos del cultivar Camarosa orgánico tuvieron mayor índice para TEAC-DPPH $(6,24 \mu \mathrm{mols}$ $\mathrm{g}^{-1} \mathrm{TE}$ ), seguido por el cultivar Camino Real orgánico $\left(6,14 \mu \mathrm{mols} \mathrm{g}^{-1} \mathrm{TE}\right)$. Los resultados obtenidos en el período cero de almacenamiento fueron próximos a los encontrados por Silva (2007) en frutos del sistema convencional durante la cosecha 2005/06, donde se observaron $5,49 \mu$ mols g $^{-1}$ TE para el cultivar Camino Real y $5,17 \mu$ mols g ${ }^{-1}$ TE para el cultivar Camarosa. Con relación a la variación porcentual de la actividad antioxidante a lo largo del período de almacenamiento, se observó un aumento superior a $16 \%$ en los frutos de los cultivares Camarosa y Camino Real, al $5^{\circ}$ día de almacenamiento (Figura 1d) en ambos sistemas de producción. Por otro lado, en el $8^{\circ}$ día de almacenamiento, se observó una disminución significativa en la actividad antioxidante $(\mathrm{p} \leq 0,05)$, principalmente para frutillas del sistema orgánico. Las variaciones en los contenidos de compuestos bioactivos $\mathrm{y}$ en la actividad antioxidante, en los sistemas de producción convencional y orgánica, están relacionadas con las diferencias en las práticas de cultivo (Wang et al., 2008).

Los coeficientes de correlación entre los valores de actividad antioxidante (TEAC) y en los niveles de fenoles totales, de antocianinas totales y de ácido ascórbico, para los dos cultivares en los dos sistemas de producción son mostrados en la Tabla 2 y Figura 2. Se observó una correlación negativa y significativa entre la actividad antioxidante y el nivel de ácido ascórbico (Tabla 2), solamente para el sistema de producción convencional. Para los tenores de antocianinas totales, los cultivares se correlacionaron positivamente con la actividad antioxidante CRC ( $\mathrm{r}=$ $0,82, \mathrm{p} \leq 0,05), \mathrm{CC}(\mathrm{r}=0,66, \mathrm{p} \leq 0,05)$, $\mathrm{CRO}(\mathrm{r}=0,84, \mathrm{p} \leq 0,05)$ y $\mathrm{CO}(\mathrm{r}=0,69$, $\mathrm{p} \leq 0,05)$. Los niveles de fenoles totales presentaron el mismo comportamiento, o sea, los dos cultivares estudiados y ambos sistemas de producción se correlacionaron positivamente con la actividad antioxidante CRC ( $\mathrm{r}=$ $0,79, \mathrm{p} \leq 0,05), \mathrm{CC}(\mathrm{r}=0,87, \mathrm{p} \leq 0,05)$, $\mathrm{CRO}(\mathrm{r}=0,72, \mathrm{p} \leq 0,05)$ y $\mathrm{CO}(\mathrm{r}=$ 
0,79, $\mathrm{p} \leq 0,05)$. Cheel et al. (2007), encontraron una correlación menor $(\mathrm{r}=0,66, \mathrm{p} \leq 0,05)$ entre fenoles totales $\mathrm{y}$ actividad antioxidante, en frutos del cultivar Chandler, a través del método DPPH. Entre el nivel de antocianinas totales y la actividad antioxidante, la correlación fue de 0,73 . Zheng et al. (2007) relataron correlación positiva entre la actividad antioxidante y el nivel de compuestos fenólicos $(r=0,96$, $\mathrm{p} \leq 0,05)$ y de antocianinas $(\mathrm{r}=0,78$, $\mathrm{p} \leq 0,05)$ en frutillas cultivar Allstar.

Los resultados demuestran que las frutillas de los cultivares Camino Real y Camarosa almacenadas a $1^{\circ} \mathrm{C}$ y 90 95\% HR, presentaron mayor pérdida de masa fresca cuando producidas en sistema orgánico.

El contenido de ácido ascórbico en frutillas disminuyó durante el almacenamiento refrigerado, independiente del sistema de producción, siendo que el sistema convencional presentó una pérdida superior al orgánico. Por otro lado, los fenoles totales, antocianinas y actividad antioxidante aumentaron durante el almacenamiento, siendo que los cultivares de producción orgánica presentaron niveles superiores para la actividad antioxidante y antocianinas. Los tenores de fenoles totales y antocianinas totales presentaron correlación positiva con la actividad antioxidante, mientras la correlación con el nivel de ácido ascórbico fue negativa.

Las frutillas producidas en sistema orgánico y refrigeradas por cinco días presentaron mayores valores de actividad antioxidante.

\section{REFERENCIAS}

AOAC - Official Methods of Analysis of AOAC International. 2000. Horwitz, W. (ed), 17. ed. Maryland: AOAC International

AYALA-ZAVALA JF; WANG SY; WANG CY; GONZÁLEZ-AGUILA GA. 2004. Effect of storage temperatures on antioxidant capacity and aroma compounds in strawberry fruit. Lebensmittel-Wissenschaft und Technologie, 37: 687-695.

BADJAKOV I; NIKOLOVA M; GEVRENOVA R; KONDAKOVA V; TODOROVSKA E; ATANASSOV A. 2008. Bioactive compounds in small fruits and their influence on human health. Biotechnology \& Biotechnological Equipment, v. 22: 581-587.
CALVETE EO; FRANCIELE MARIANI F; WESP CL; NIENOW AA; CASTILHOS T; CECCHETTI DEF. 2008. Fenologia, produção e teor de antocianinas de cultivares de morangueiro em ambiente protegido. Revista Brasileira de Fruticultura, 30: 396-401.

CANTILLANO RFF; BENDER JR; LUCHSINGER LL. 2003. Fisiologia e manejo pós-colheita. In: CANTILLANO RFF. Morango. Pós-colheita. Brasília:Embrapa Informação Tecnológica. p. 14-24. (Frutas do Brasil;42).

CANTILLANO RFF. 2008. Produção integrada de pêssegos: manuseio pós-colheita e logística. Pelotas: Embrapa Clima Temperado, 41 p. Embrapa Clima Temperado. Documentos, 228.

CANTILLANO RFF; CASTAÑEDA LMF; TREPTOW RO; SCHUNEMANN APP. 2008. Qualidade físico-química e sensorial de cultivares de morango durante o armazenamento refrigerado, Embrapa Clima Temperado. Boletim de Pesquisa e Desenvolvimento, 75: 31

CASTRO RL; CASALI VWD; BARRELLA TP; SANTOS RHS; CRUZ CD. 2003. Produtividade de cultivares de morangueiro em sistema de cultivo orgânico. Horticultura Brasileira 21: 227-230.

CHEEL J; THEODULOZ C; RODRYGUEZ JA; CALIGARI PDS; SCHMEDA-HIRSCHMAN G. 2007. Free radical scavenging activity and phenolic content in achenes and thalamus from Fragaria chiloensis ssp. chiloensis, F. vesca and F. x ananassa cv. Chandler. Food Chemistry 102: 36-44.

CROZIER A; BURNS J; AZIZ AA; STEWART AJ; RABIASZ HS; JENKINS GI; EDWARDS CA; LEAN MEJ. 2000. Antioxidant flavonols from fruits, vegetables and beverages: measurements and bioavailability. Biology Research, 33: 79-88.

ERLUND I; KOLI R; ALFTHAN G; MARNIEMI J; PUUKKA P; MUSTONEN P; MATTILA P; JULA A. 2008. Favorable effects of berry consumption on platelet function, blood pressure, and HDL colesterol. American Journal of Clinical Nutrition, 87: 323-31.

FERREYRA SRM; VIÑA Z; MUGRIDGE A; CHAVES AR. 2007. Growth and ripening season effects on antioxidant capacity of strawberry cultivar. Scientia Horticulturae, 112: 27-32.

HERNANDEZ-MUÑOZ P; ALMENAR E; OCIO MJ; GAVARA R. 2006. Effect of calcium dips and chitosan coatings on postharvest life of strawberries (Fragaria x ananassa). Postharvest Biology and Technology, 39: 247-253.

HOLCROFT DM; KADERAA. 1999. Controlled atmosphere-induced changes in $\mathrm{pH}$ and organic acid metabolism may affect color of stored strawberry fruit. Postharvest Biology and Technology, 17: 19-32.

KALT W; FORNEY CF; MARTIN A; PRIOR RL. 1999. Antioxidant capacity, vitamin C, phenolics, and anthocyanins after fresh storage of small fruits. Journal of Agricultural and Food Chemistry, 47: 4638-4644.

KROLOW ACR; SCHWENGBER JE; FERRI
N. 2007. Avaliações físicas e químicas de morango cultivar. Aromas produzidos em sistema orgânico e convencional. Revista Brasileira de Agroecologia, 2: 1732-1735.

LEES DH; FRANCIS FJ. 1972. Standardization of pigment analyses in cranberries. HortScience, 7: 83-84.

MALGARIM MB; CANTILLANO RFF; COUTINHO EF. 2006. Sistemas e condições de colheita e armazenamento na qualidade de morangos cv. Camarosa. Revista Brasileira de Fruticultura 28: 185-189.

MAZARO SM; DESCHAMPS C; MIO LLM; BIASI LA; GOUVÊA A; SAUTTER CK. 2008. Comportamento pós-colheita de frutos de morangueiro após a aplicação pré-colheita de quitosana e acibenzolarsimetril. Revista Brasileira de Fruticultura 30: 185-191.

NACZK M; SHAHIDI F. 2004. Extraction and analysis of phenolics in food. Journal of Chromatography. 1054: 95-111.

NUNES MCN; BRECHT JK; MORAIS AMMB; SARGENT S. 1998. Controlling temperature and water loss to maintain ascorbic acid levels in strawberries during postharvest handling. Journal of Food Science 63: 1033-1036.

NUNES MCN; BRECHT JK; MORAIS AMMB; SARGENT S. 2005. Possible influences of water loss and polyphenol oxidase activity on anthocyanin content and discoloration in fresh ripe strawberry (cv. Oso Grande) during storage at $1{ }^{\circ} \mathrm{C}$. Journal of Food Science 70 : 79-84.

OLSSON ME; ANDERSSON SC; BERGLUND RH; GUSTAVSSON KE. 2007. Extracts from organically and conventionally cultivated strawberries inhibit cancer cell proliferation in vitro. Acta Horticulturae 1: 189-194.

OZGEN M; REESE RN; TULIO JUNIOR AZ; SCHEERENS JC; MILLER AR. 2006. Modified 2,2-azino-bis-3ethylbonzothiazoline-6-sulfonic acid (ABTS) method to measure antioxidant capacity of selected small fruits and comparison to ferric reducing antioxidant power (FRAP) and 2,2'-diphenyl-1-picrylhydrazyl (DPPH) methods. Journal of Agricultural Food Chemistry 54: 1151-1157.

PELAYO-ZALDÍVAR C; EBELER SE; KADER AA. 2005. Cultivar and harvest date effects on flavor and other quality attributes of California strawberries. Journal of Food Quality 28: 78-97.

REGANOLD JP; ANDREWS PK; REEVE JR; CARPENTER-BOGGS L; SCHADT CW; ALLDREDGE JR; ROSS CF; DAVIES NM; ZHOU J. 2010. Fruit and soil quality of organic and conventional strawberry agroecosystems. PLoS One 5: 12346.

SEERAM NP; ADAMS LS; ZHANG Y. 2006. Blackberry, black raspberry, blueberry, cranberry red raspberry, and strawberry extracts inhibit growth and stimulate apoptosis of human cancer cells in vitro. Journal of Agricultural and Food Chemistry 54: 93299339.

SILVA RS. 2007. Potencial antioxidante correlacionado com fenóis totais e antocianinas de cultivares de amora-preta, mirtilo, morango e pêssego. Pelotas: UFPEL. 
60p (Dissertação mestrado).

SINGLETON VL; ROSSI JA. 1965. Colorimetry of total phenolics with phosphomolybidicphosphotungstic acid reagents. American Journal of Enologie and Viticulture, 16: 144-158.

STASOFT Inc. 1996. STATISTICA for Windows (Computer program manual). Tolsa, OK: StatSoft, Inc., 2300 East 14 th Street, Tulsa, OK 74104, phone: (918) 749-1119, fax: (918) 749-2219, e-mail: info@statsoftinc.com, WEB: http:// www.statsoftinc.com.

STRECK EV; KÄMPF N; DALMOLIN RSD; KLAMT E; NASCIMENTO PC;
SCHNEIDER P; GIASSON E; PINTO LFS. 2008. Solos do Rio Grande do Sul. 2.ed. Porto Alegre, EMATER/RS-ASCAR, 222p.

TERRAZZAN P; AGUILA JS; HEIFFIG LS; KLUGE RA. 2006. Physicochemical characterization of refrigerated strawberries conventional and organic crop systems. Revista Ibero-Americana de Tecnologia Postcosecha, 8: 33-37.

TORRES UC; GUZMÁN JL; MOORECARRASCO R; PALOMO GI. 2008. Efecto antitrombótico, una característica poco conocida de las frutas y hortalizas. Revista Chilena de Nutrición 35: 10-17.

WANG SY; CHEN CT; SCIARAPPA W;
WANG CY; CAMP MJ. 2008. Fruit quality, antioxidant capacity, and flavonoid content of organically and conventionally grown blueberries. Journal of Agricultural and Food Chemistry 56: 5788-5794.

WINKEL-SHIRLEY B. 2001. Flavonoid biosynthesis. A colorful model for genetics, biochemistry, cell biology, and biotechnology. Plant Physiology, 126: 485-493.

ZHENG Y; WANG SY; WANG CY; ZHENG W. 2007. Changes in strawberry phenolics, anthocyanins, and antioxidant capacity in response to high oxygen treatments. Lebensmittel-Wissenschaft und Technologie, 4: $49-57$ 